\title{
The Pleiades question, the definition of the zero-age main sequence, and implications
}

Floor van Leeuwen

Institute of Astronomy, University of Cambridge, Madingley Road, Cambridge, CB3 0HA, UK email: fvl@ast.cam.ac.uk

\begin{abstract}
Determinations and measurements of the parallax of the Pleiades, obtained with ground-based studies and with Hipparcos data, are reviewed. A number of uncertainties in both sets of data are found. Although a further correction for abscissa correlations brings the Hipparcos determination closer to the ground-based value, the difference still seems too large. The new Hipparcos parallax determinations seem to reinforce a possible age related effect. A new reduction of the Hipparcos data is in progress. It reduces significantly the contribution of the attitude noise, providing higher accuracies and lower correlation levels for the brighter stars.
\end{abstract}

\section{Introduction}

The Pleiades cluster is the oldest recorded 'mini' constellation on the sky. In many ancient civilizations it played a crucial role in defining the seasons, and in today's astrophysics it has led the way to a number of crucial discoveries: the colour-magnitude relation by Hertzsprung (1915), for example, was based on his study of the Pleiades and Hyades stars; the discovery in 1980 of very fast rotation of $\mathrm{G}$ and $\mathrm{K}$ dwarfs shortly before becoming proper main sequence stars was first made in the Pleiades cluster (van Leeuwen \& Alphenaar 1982; Stauffer et al. 1984; van Leeuwen et al. 1987). And now there is the Hipparcos parallax determination for the cluster, which seems to be at odds with the expected value by quite a margin (van Leeuwen 1999a; Robichon et al. 1999).

It should be no surprise that it is so often the Pleiades cluster that leads the way, or even causes problems. It is the same reason it had been so much noticed by ancient civilisations: the cluster stands out among the stars in the Northern hemisphere. It just so happened to fit nicely on a standard photographic plate size for an astrographic refractor, which made it a very suitable early candidate for proper motion studies (see, for example, Hertzsprung 1947; Vasilevskis et al. 1979). On the other hand, it is not too dense to generally cause problems in observations (unlike the Trapezium Cluster in Orion or most globular clusters), though it is situated in an area of dust clouds, showing as reflection nebulae and very significant variations in reddening for individual stars (van Leeuwen et al. 1986).

Since the first publication of the Hipparcos parallax determination for the cluster (van Leeuwen \& Hansen-Ruiz 1997) many claims and counter claims have been produced on its validity. The value presented in that paper has since been revised downwards, on the basis of a better handling of the abscissa correlations in the data (van Leeuwen 1999a; Robichon et al. 1999). The arguments of both sides have generally not met: from the Hipparcos side it was claimed that aspects of the data reduction and catalogue construction, together with statistical tests performed on the reduced data, give very little leeway to account for the presence of widespread systematic errors in the published parallaxes (van Leeuwen 1999b, 2000) as was claimed to be the case by, for example, Narayanan \& Gould (1999). On the other hand, models of stellar structure and stellar evolution seem to indicate that the differences between the positions of the main sequences for the Pleiades and 
other open clusters as based on the Hipparcos parallaxes are too large (see, for example, Pinsonneault et al. 2004, and references therein), although some authors claim from similar data that this may not be the case (Robichon et al. 2000; Castellani et al. 2002; Percival et al. 2003).

So how may we tackle this problem? In as far as the Hipparcos data are concerned the only way of tackling it is by going back to the original data and looking at all the individual steps and assumptions made along the way of the data reductions. That is a very big job, which has already taken several years (van Leeuwen \& Fantino 2003). For all determinations that implement models, it is worthwhile to re-consider the accuracies and intrinsic (cosmic) noise levels associated with the calibrations on which they are calibrated. If data from the Pleiades cluster are used to calibrate model parameters, those model parameters should not be used to calibrate the distance of the cluster. Assumptions used in deriving reddening should be looked into: when reddening has been derived through shifting the data to an isochrone in the colour magnitude diagram, it is no longer an independent parameter. Reddening should only be derived through independent methods, such as colour-colour diagrams (although even there one has to be very careful) and polarization measurements. The uncertainty in the absorption needs to be fully taken into account. For example, the Pleiades are affected by differential reddening, and the most seriously affected star (HII 1084, situated near the Merope nebula) shows quite clearly deviating reddening directions in the two-colour diagrams of the Walraven photometry (van Leeuwen et al. 1986), indicating local, non-average dust properties.

It would be of interest not just to look at the situation for individual clusters, but also to consider the overall behaviour of the 10 clusters for which reliable parallaxes are now available: does the Pleiades problem stand on its own, or is it part of a more general feature? Discrepancies such as those observed for the Pleiades distance should be used as a motivation to reconsider critically ALL aspects from both sides of the medallion: the correct understanding of why a result is wrong is in the end far more relevant than the claim that someone else's result is wrong because it differs from your result, and you know you don't make any errors. Reading through the various claims and counter-claims it becomes clear that an expected result is not always a reliable result, and maybe an unexpected results is not necessarily a wrong result.

This paper consists of two main parts. The first considers aspects of ground-based distance determinations for the Pleiades cluster, looking at photometric methods, convergent point determinations, ground-based parallax determinations and double star analysis. Section 2 considers the Hipparcos data, with impressions from a completely new reduction of the data and looking at methods proposed to correct cluster parallaxes from the published data. As the new reduction is not finished yet, and within the Hipparcos tradition we do not make available preliminary results, it is too early at this stage to present any other conclusion than a general suggestion: be critical, not only to unexpected results, but also to studies that seem to confirm expected results so very well.

Hipparcos-based parallax values for open clusters as used in this paper are based on a revised analysis of the published data, which includes a better accounting for the differences in abscissa correlations for stars of different magnitudes. This correction, which appears to bring some Hipparcos results in closer agreement with ground-based results (giving a provisional value for the Pleiades parallax of 8 mas), is, however, not well determined. 


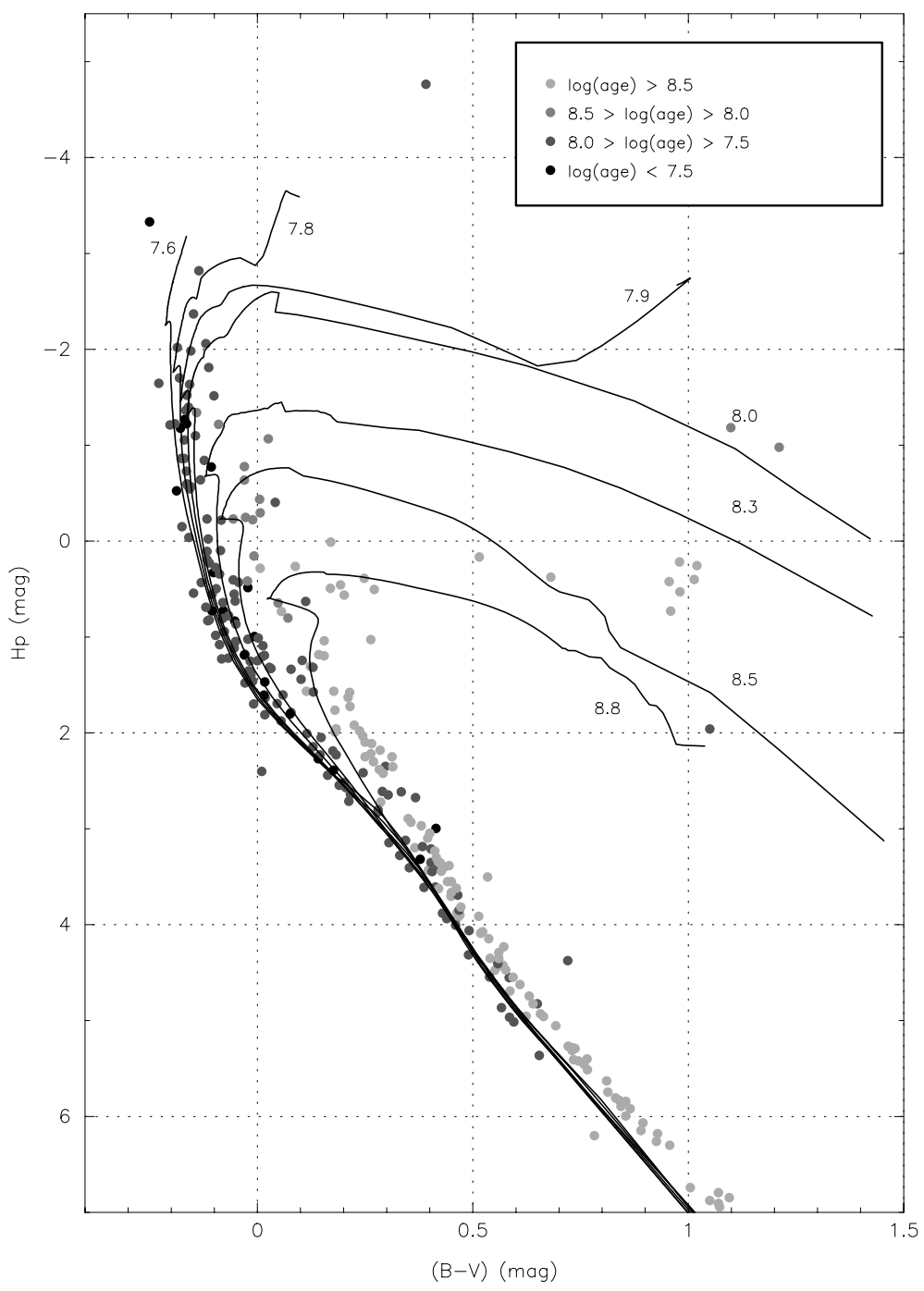

Figure 1. The combined HR diagram for 10 open clusters with Hipparcos parallaxes in a grey-scale according to cluster age. Reddening corrections have been applied. The differences between the young and the old clusters appear to start earlier than indicated by the isochrones (courtesy of Demarque \& Kozhurina-Platais).

\section{Ground-based studies}

\subsection{Photometric determinations}

A photometric distance determination assumes a position for the main sequence (given an assumed or measured metallicity) and derives for that position a distance modulus; after correcting for reddening a distance is obtained. Often the reddening correction is included in the fitting procedure. As has been recognized by numerous authors before, it is not possible to use this method to detect an actual difference between the position of the Pleiades main sequence and mean main sequence to which it is calibrated: the assumption of the method is that there is no difference. For the same reason, all these determinations end up with nearly the same value for the distance modulus of the cluster, equivalent to a parallax of $7.59 \pm 0.14$ mas (see, for example, Pinsonneault et al. 1998). 
For establishing a difference with the Hipparcos value these determinations are of rather limited value: you don't disprove a discrepancy by assuming that it doesn't exist.

Turning the argument around, it becomes the key issue: the relative positions of open cluster main sequences can provide essential information on calibration of models of stellar structure, evolution, and stellar atmospheres. Only a determination completely independent of these models can do so. Fig. 1 shows one way of looking at this problem. The photometric data, corrected for reddening, are shown for 10 open clusters with parallax determinations: although there may be a small discrepancy between the young (IC-2602, IC-2391, Pleiades) and the old clusters (Hyades, Coma Ber), there also appears to be good agreement within these age groups. The excess in blue for the faintest Pleiades stars is most likely due to chromospheric activity of these still relatively young objects (see also van Leeuwen et al. 1986; Percival et al. 2003; Stauffer et al. 2003).

\subsection{Convergent point determination}

The Pleiades cluster is poorly suited for a convergent point determination (CPD) such as used in the past for the Hyades cluster. The CPD compares the apparent contraction or expansion of the cluster, as observed from the proper motions of its members, with its radial velocity to derive a cluster distance. It relies on absolute proper motions (to enable measuring the expansion), a wide spread of cluster stars on the sky (to make the expansion large enough to obtain a significant result), and a large radial velocity (to produce a substantial expansion), and the Pleiades has none of these qualities. The radial velocity is only about $5.7 \mathrm{~km} \mathrm{~s}^{-1}$, which gives an expansion-related proper motion at $2^{\circ}$ from the cluster centre of only $0.23 \mathrm{mas} \mathrm{yr}^{-1}$. Beyond $2^{\circ}$ the density of cluster members is very low, and within $2^{\circ}$ the effect is even smaller. These effects are also substantially smaller than the intrinsic velocity dispersion in the cluster (van Leeuwen 1984), which makes them very hard to determine accurately enough to provide a reliable distance estimate.

There are three papers referring to such a CPD for the Pleiades. One, by Madsen et al. (2002), shows that the expected error of a CPD for the Pleiades is considerably larger than the determination based on the parallaxes. Narayanan \& Gould (1999) determined a CPD parallax of $7.5 \pm 0.6$ mas, which is at $1 \sigma$ from the published Hipparcos value (less than $0.8 \sigma$ from the new determination), even though the authors claim this to be a significant difference. Finally there is the study by Zhao Junliang and Li He, who claimed to have derived a parallax of $7.4 \pm 0.4$ mas for the cluster. These authors, however, used for their determination the differential proper motions as published by Hertzsprung (1947), which do not contain the necessary information for a CPD. Also, they used only a $2^{\circ}$ diameter field and radial velocities for 63 stars. The radial velocity effects in such a small field are at a level of a $1 \mathrm{~ms}^{-1}$ and less, well below anything detectable. A year after the publication of their study, one of the authors confirmed (private communication) that substantial errors had been made in this study (the CPD studies were referred to by Makarov (2002) as 'conclusive evidence' that there are problems with the Hipparcos parallax determinations in some parts of the sky). For the time being it seems to be clear that no sufficiently accurate CPD parallax for the Pleiades cluster can be expected to either confirm or contradict the Hipparcos result.

\subsection{Ground-based parallaxes}

The most recent ground-based determination of the Pleiades parallax is by Gatewood et al. (2000), who give a value of $7.64 \pm 0.43$ mas for the mean parallax of 18 cluster members. Ground-based parallaxes are always differential, which means that they rely on assumptions made about the distances of the background stars that are used as 


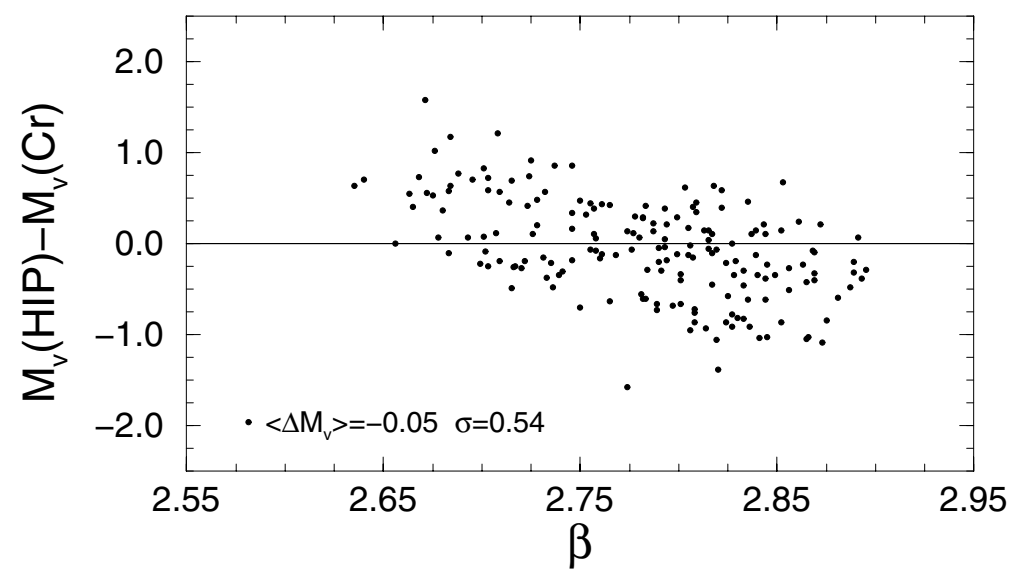

Figure 2. Differences between parallax- and photometry-based absolute magnitudes. The spread is due to several contributions: less than $0.2 \mathrm{mag}$ from uncertainties in parallaxes, errors in colour indices, inaccuracies in photometric calibrations, rotation, cosmic dispersion (from Jordi et al. 2002.)

a reference frame. In Gatewood et al.'s study an iterative method is used in which the starting values of these distances (in the form of the assumed spectral types and observed apparent magnitudes) are adjusted until an acceptable solution is obtained. The difference with the current Hipparcos parallax determination for the Pleiades is less than $1 \sigma$ and therefore not significant.

\subsection{Double stars}

Although double star determinations are often presented as the alternative to parallax measurements, in that they provide a direct measure of the distance of a system, this only applies if the determination is based purely on measurements of both orbital motions and radial velocities. For those Pleiades stars that have a known orbit, the radial velocities are difficult to measure with sufficient accuracy due to the rapid rotation and sparsity of spectral lines of the stars involved, and for those with a radial velocity curve no astrometric orbit data are available. Calculations based on only either orbital motion or radial velocity measurements still rely on stellar models. Two recent determinations (Pan et al. 2004; Munari et al. 2004) give distance estimates for two binaries in the Pleiades: HD 23850 (7.41 \pm 0.11 mas) and HD 23642 (7.58 \pm 0.11 mas). Both values are within 1 to $1.5 \sigma$ from the determinations for these stars by Hipparcos. With their dependence on stellar-model parameters it seem premature to draw far-going conclusions from the difference with the parallax derived from the Hipparcos data. In particular, it is incorrect to quote these parallax values with the same formal errors as measurements of the cluster distance. When used to assess the cluster distance, the dispersion of parallaxes of cluster members along the line of sight has to be added to the formal error. As a comparison one may take the distribution of Pleiades stars on the sky, for which the projected centre is quite poorly determined (to a level of about $1 \mathrm{pc}$ ).

\subsection{Cosmic spread in stellar parameters}

In deriving distances from double star data as described above, assumptions are made about the reproduceability of stellar parameters as derived from observational quantities such as colour indices or spectral types. With the publication of the Hipparcos data it became possible to re-examine many of the basic calibrations of photometric systems and spectroscopic determinations, in particular where they concerned absolute magnitudes 
and their dependence on chemical composition (see, for example, Gomez et al. 1997, 1998; Jordi et al. 2002; Grenon 2002). A considerable cosmic dispersion is observed for photometric distance-modulus calibrations (see Fig. 2). It therefore seems appropriate to exercise some care when applying such calibrations to derive stellar parameters: whenever we are able to observe with sufficient detail the calibration relations appear to apply only globally, showing substantial deviations for individual objects. Application of old, pre-Hipparcos calibrations also generally introduces systematic errors in the estimated parameters (see, for example, Jordi et al. 2002).

\subsection{The lack of spread in parallax determinations for the Pleiades}

The opposite is observed for ground-based determinations of the Pleiades parallax or distance modulus: values produced in various studies generally agree very much better with the 'established value' than one would expect on the basis of the stated formal errors (see some of the figures quoted above), even when derived from completely unsuitable data.

\section{The Hipparcos data}

\subsection{The data}

The Hipparcos data have been published in great detail, allowing studies at a level that only very few would pursue (ESA 1997; van Leeuwen 1997). It has made possible the studies by, for example, Knapp, Pourbaix \& Jorissen (2001), Knapp et al. (2003), Pourbaix \& Jorissen (2000) and Pourbaix \& Boffin (2003) on very red variables, where epoch information on the $V-I$ indices have led to more accurate astrometric parameters for these stars. Cluster parameter solutions whereby a single solution for the cluster parallax and proper motion is obtained from the abscissa measurements of the member stars (van Leeuwen \& Evans 1998) is an other example of the use of these data. Allowing this has always been the intention of the publication of the Hipparcos data: within the time limits set for the publication of the data not all aspects could be fully investigated, but sufficient data should be provided to make a posteriori corrections possible. Still, to respond to some of the suggestions made as to what may be wrong with the cluster parallaxes, or even the general reliability of parallax data in the Hipparcos catalogue, it was required to refer back to the original, approximately 112 GByte of raw data. Only one, nearly complete, copy is still in existence: the data files used as input for the reductions by the Northern Data Analysis Consortium (NDAC) (Lindegren et al. 1992). A sufficiently detailed set of intermediate files still exists for the reductions of the FAST (Kovalevsky et al. 1992) consortium. Detailed analysis implies rewriting large amounts of software for a more modern hardware environment, and allowing more interactive control over the reduction processes.

In many ways reductions have also become much simpler. As a comparison, the original data arrived on nearly 1200 9-track tapes, filling a small office. In the NDAC consortium these data were transferred to optical disks, from which the data reductions were performed, mainly overnight and during weekends. One pass through all the data took several months. In the new application, the data are kept on 24 DVDs, and reductions are done on a laptop computer, taking two to three weeks to process all mission data. A complete set of new software is implemented to analyse the data for single stars, to provide input for double star analysis, and to provide a multitude of statistical tests and other data quality controls. Results reported below on data quality are mostly obtained with this software as applied on the raw data (see also van Leeuwen \& Fantino 2003). 


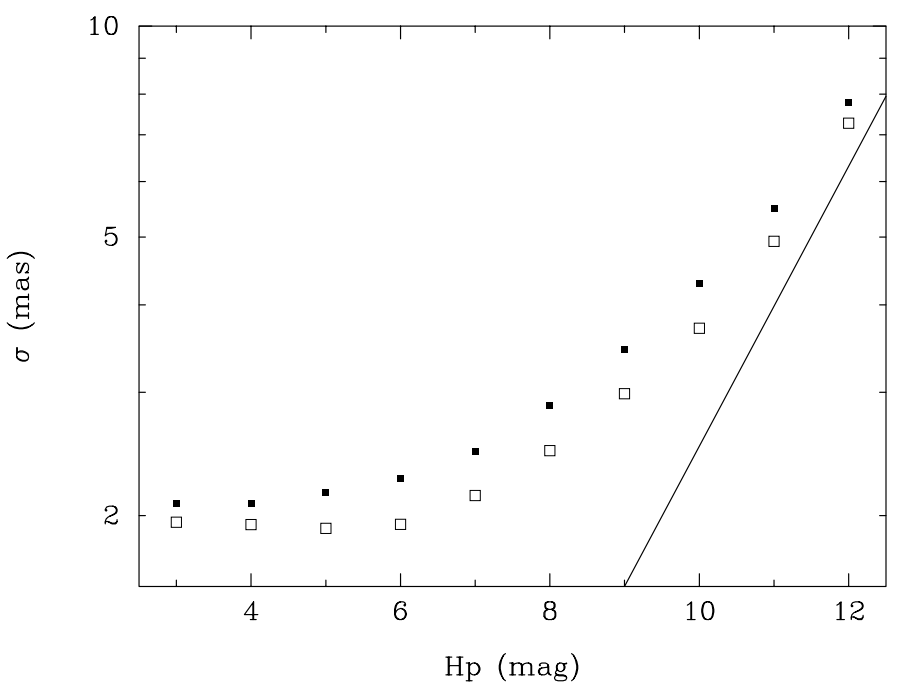

Figure 3. Standard deviations ( $\sigma$ in mas) for the abscissa residuals as a function of the $H p$ magnitude. Open symbols refer to FAST data, filled symbols to NDAC data. The diagonal line represents the expected relation for Poisson noise from the photon counts, given one and the same integration time for all intensities. The flattening of the relation towards brighter magnitudes shows the 2 mas noise contribution from primarily the along-scan attitude modelling contributions (from van Leeuwen 1997).

The data are subdivided into data sets, covering data collected over one orbit of the satellite, just under 11 hours. The mission results are based on the data collected for nearly 2300 data sets.

\subsection{One-dimensional astrometry}

The Hipparcos measurements provide one-dimensional positions: transit times of stellar images as measured on a modulating grid while the fields of view of the telescope describe a great circle on the sky. These one-dimensional measurements are referred to as abscissae or transit times. In fitting the along-scan attitude (rotation phase) of the satellite, the abscissa residuals for both fields of view are fitted as a function of time. The remaining abscissa residuals for individual stars are collected and averaged per field transit, and then fitted as a function of the five astrometric parameters (position, proper motion and parallax), relative to an a priori solution. Because of the relatively large errors on the $a$ priori astrometric parameters of the program stars, these two parameter sets initially had to be estimated in one solution, referred to as the Great-Circle reduction (van der Marel \& Petersen 1992). In the new solution this is no longer required, allowing improvements in the stability and accuracy of the along-scan attitude solution. By scanning each image in at least two different directions every 6 months, and accumulating data over 3.5 years, corrections to all five astrometric parameters can be solved.

The noise on the abscissa residuals contains two main contributions: attitude-fitting and Poisson noise. The first component dominates for bright stars, the second for faint stars. The attitude noise is more or less constant, though it will usually be somewhat higher in areas of the sky with few bright stars. In the published data, the effects of attitude noise start to get noticed for stars at 9 th magnitude and brighter (see Fig. 3). In the new reduction the attitude noise level has been much reduced, and becomes noticeable only at 4th magnitude and brighter (van Leeuwen \& Fantino 2003, and see below). 


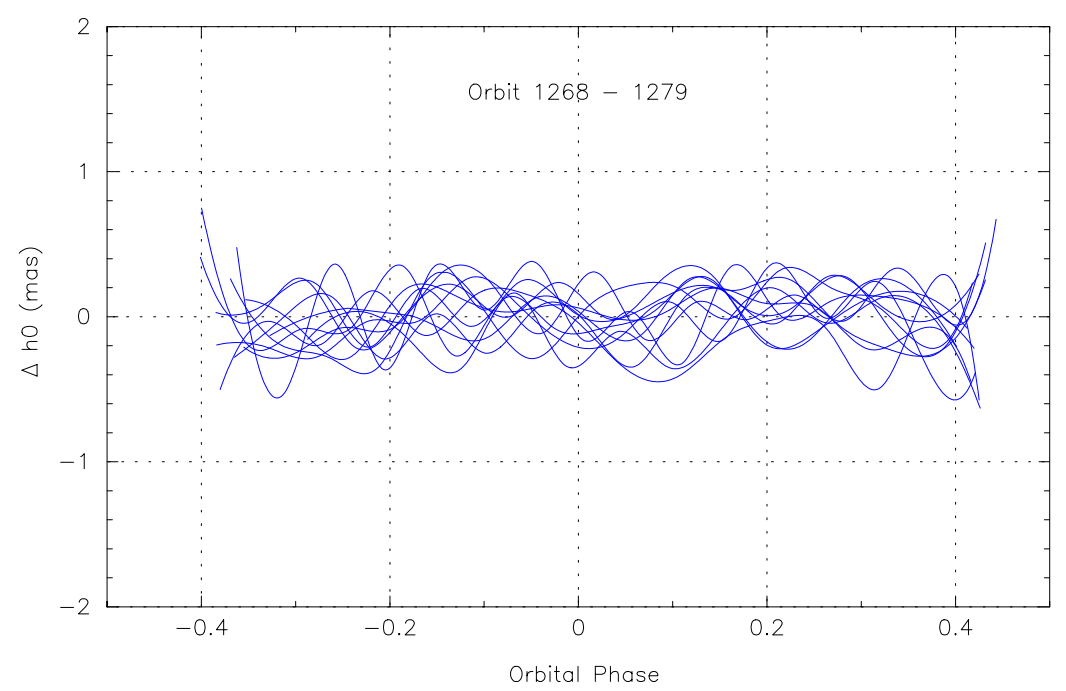

Figure 4. Noise levels on the reconstruction of the basic angle as observed from fitted residuals in the preceding and following fields of view. The data are for 12 successive orbits from May 1991. The rotation phases of successive circles at a given orbital phase change very little from one orbit to the next (from van Leeuwen \& Fantino 2003).

\subsection{Parallax errors and basic-angle stability}

The basic angle of the Hipparcos telescope defines the angular distance on the sky between the projections from the two fields of view. The stability of the basic angle was a paramount condition for the success of the mission. In particular, a modulation of the basic angle with the rotation of the satellite could introduce a zero-point error in the parallaxes, and, it was thought, any systematic error in parallaxes locally on the sky had to be associated with systematic basic angle variations. Variations in the basic angle can be observed from systematic differences between the abscissa residuals in the two fields of view. No systematic modulation of the basic angle has been observed for $99 \%$ of the mission (see Fig. 4), but what was discovered is the presence of systematic drifts in the basic angle for $1 \%$ of the data sets (van Leeuwen \& Penston 2003). Most of these drifts had not been detected for the preparation of the published data. All can be identified with known events of the mission. The most common of these was not identified as an anomaly, but could still be traced back in the mission reports. It was due to a communication problem between the on-board computer and the ground station, which could only be solved by turning off the entire payload. Unfortunately, this included the temperature control of the payload, and when it was switched on again, the change in temperature was noted from the value of the basic angle. The adjustment to a stable, controlled value usually took only a few hours. In the new analysis these drifts are solved for and corrected in the reduced data, except for secondary effects on the scale and rotation in the field of view.

Most importantly, no systematic modulations of the basic angle are observed down to a level of at least 0.1 mas. This confirmed earlier tests on the parallax zero-point, which was found to be less than 0.1 mas by Lindegren (1995); Arenou et al. (1995). This corresponds to a modulation level below 0.035 mas. Thus, there is no way in which lack of stability of the basic angle could have caused a difference of 0.5 to 1.0 mas in the parallax of the Pleiades cluster. In addition, as was shown by van Leeuwen (1999b), the accumulation of systematic errors over the $8^{\circ}$ to $10^{\circ}$-diameter field of the Pleiades cluster is quite inefficient. 


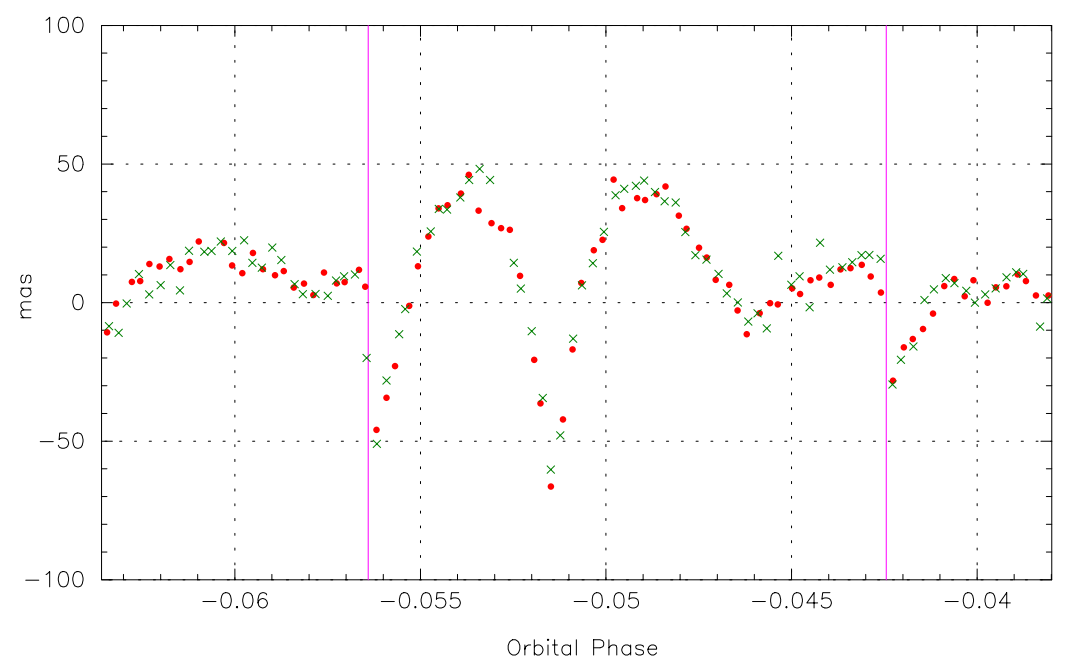

Figure 5. An example of an external hit of the satellite as recorded in the abscissa residuals before the final along-scan attitude fitting. The hit took place around phase -0.0515 , and resulted in a change in the rotation velocity of the satellite by $4.7 \mathrm{mas} \mathrm{s}^{-1}$. Hits like these took place on average about once every two weeks. In the old solution such data were usually not recognized, in which case it would produce strongly correlated attitude errors for the data involved, or the data would be thrown away. In the new solution the discontinuity in the rate is fully accommodated and only a few seconds worth of data are lost (due to the uncertainty of exactly positioning the time of the hit).

\subsection{Data disturbances}

Other disturbances of the data have been noted too, but the amount of data affected is again small. The two main effects are external hits of the satellite (at a rate of roughly one every two weeks for an impact detection level of 7 to $8 \mathrm{~nJ}$ or higher, see Fig. 5), and scan phase discontinuities at times of thermal adjustments of the spacecraft, primarily around eclipses (see Volume 2, Chapter 11, in ESA 1997). Both of these effects were known to exist and had in some cases already been removed from the data by cutting out affected time intervals. There are now an estimated 1500 phase discontinuities recorded over the mission, affecting up to $1.5 \%$ of the data (either by it being thrown away or, at some stage, iterated out). In the new reduction the vast majority of these events are identified and accommodated in the reduction such that only about $0.01 \%$ of the data are lost. These effects, when not properly accounted for or removed, will have increased locally the noise level on the data, which may not always have been recognized.

\subsection{Statistical properties of the Pleiades parallax determination}

One of the problems we have had with accepting the idea that the Hipparcos determination for the Pleiades parallax is wrong comes from the statistical properties of the parallax solution. This solution is not based on just averaging the observed parallaxes of the probable members, but is an actual solution of the cluster parallax and proper motion based on the individual abscissa residuals for cluster members (van Leeuwen \& Evans 1998). This solution takes into account correlations between the FAST and NDAC data and between abscissae measured in the same data set. The correlation levels have now been adjusted to account for differences for stars of different brightness, which brings the parallax to approximately 8.0 mas, back at the value that has been used for many decades. However, this value is sensitive to the exact implementation of the correlation levels for the bright stars, which are impossible to derive with great accuracy. In the new 
solution these correlation levels will be much reduced, as in general the attitude noise is lower by about a factor three. The current Pleiades parallax is based on 2246 abscissae for 56 stars, originating from 133 different data sets, spread over the entire mission. The stars occupy an area of $10^{\circ}$ diameter on the sky. The unit-weight standard deviation of this solution is 1.08, which doesn't seem to indicate the presence of serious shortcomings in the solution, though there are indications that not all is well with the solution. These indications come from the proper motions rather than the parallaxes. The spread of the proper motions is too high, and those for the centre of the field fit poorly with ground-based studies of much higher precision (Vasilevskis et al. 1979).

\subsection{Absolute and relative parallaxes}

Not everything is always the way it looks. Makarov $(2002,2003)$ presented a method for correcting cluster parallaxes using stars observed simultaneously in the other FOV. In doing so he made several assumptions, some of which are impossible to verify from the published data, but can be verified from the raw data. Makarov applied two arguments for his study which contradict each other: he assumed that the apparent discrepancies in the Pleiades parallax are the result of the group being more or less detached from the catalogue, which may well be, at least partly, the correct explanation. But this situation would, for the same reason, not allow the use of stars from the other FOV to apply abscissa residual corrections, as the data for these stars is assumed to be poorly connected. On the other hand, the assumptions made on the way the measurements of these coinciding stars can be used to correct the Pleiades abscissae require an optimal connectivity of this field with the rest of the catalogue, which would make a correction unnecessary. The unsuitability of these data for a correction as applied by Makarov had already been pointed out in van Leeuwen (1999b).

The method as applied by Makarov results, amongst others, in transferring photon noise from transits in the other field of view on the abscissa measurements of the cluster stars, and will generally make those measurements worse (producing an 'expected' result is not a validation for the method). It was observed from the raw data that in the majority of cases the measurements of the stars in the other field of view are dominated (in amount of observing time assigned) by those not coinciding with the cluster stars. This confirms that the data for some cluster centres may not be optimally attached to the catalogue, possibly allowing for significant, local parallax zero-point offsets. How this affected the results will be different for the FAST and NDAC reductions, and as the published catalogue presents an averaged result, the final effect may not be very strong. In order to check the possible impact of this effect, the connectivity for each single star in the catalogue was determined, producing a map of potential soft spots for all those areas where the weight may have been generally higher than for most of the coinciding data in the other field of view. A comparison of this map with a map of the differences between the FAST and NDAC solutions shows a few small areas among the Southern OB associations with differences at the $2 \sigma$ level and above. The Pleiades does show strongly in the 'soft spots' map, but not in the differences between FAST and NDAC. Neither solution will bring the Pleiades parallax down to a level of 7.3 to 7.7 mas. Also, the halo of the cluster is unaffected, and shows a parallax of around 8.0 mas. Thus, although it is likely that the centre of the Pleiades cluster was not solidly attached to the catalogue, the impression at this stage is that the error is probably no more than 0.2 to 0.4 mas.

The potential influence of the weight disparities between the two fields of view has been taken into account for the new reduction, where corrections are applied to reduce large disparities. The dynamical modelling of the attitude in the new solution, as well as 


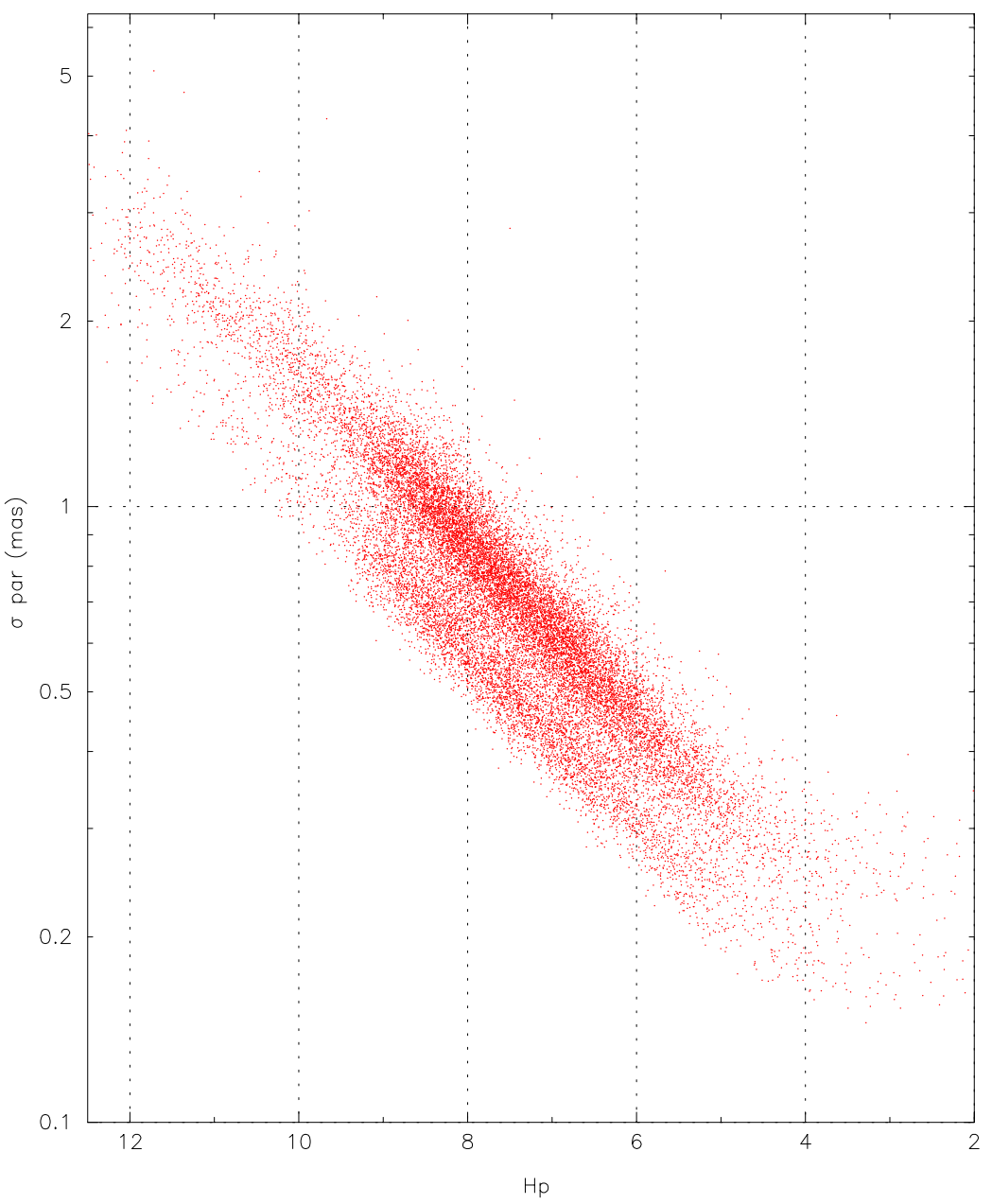

Figure 6. Formal errors on parallax determinations in the new solution. A significant reduction in the noise on the along-scan attitude reconstruction ensures that accuracies for a much larger fraction of the stars are dominated by Poisson statistics. Accuracies for the brightest stars have improved as a result by about a factor three. The bimodal character of the formal errors is due to the scanning strategy, and reflects different ecliptic latitudes.

the decoupling between the attitude and abscissa corrections also adds to reducing these effects.

\subsection{The new reduction}

The new reduction of the Hipparcos data is well on its way. It is based on a dynamic modelling of the satellite attitude and takes care of various other problems in the data. Preliminary results show an improvement in the attitude noise by a factor three or more, leading to a reduction in the errors of the astrometric parameters for bright stars by a factor 3 (see Fig. 6). This is currently only a measure of precision. A reduction in the number and size of negative parallaxes among the brighter stars indicates that at least some of this is indeed an improvement in accuracy. Determining by how much exactly the accuracies have improved is still one of the major tasks ahead. The publication of these data and the methods used to derive them is planned in the form of a book, describing the use of the data as well as aspects of how the data has been derived. As with the 
original publication of the Hipparcos data, there will be no release of preliminary results, and the first results of the new reduction are expected towards the end of 2004. This is likely to include new parallax determinations for the open clusters, much less affected by the uncertainties caused by abscissa-error correlations and weight disparities.

\section{Conclusions}

Problems with the Hipparcos data for some open cluster centres can most likely be attributed to high correlations in abscissa residuals for bright stars and possibly to weight disparities between two fields of view in the along-scan attitude reconstructions. A provisional adjustment of the abscissa error correlation levels for bright stars produces a reduction of the parallax to a value of around 8 mas, but the exact level of these corrections is difficult to determine. In the new solution the correlations are expected to play a much smaller role.

\section{References}

Arenou, F., Lindegren, L., Froeschlé, M., Gómez, A. E., Turon, C. Perryman, M. A. C., \& Wielen, R. 1995, A\&A, 304, 52-60

Castellani, V., Degl'Innocenti, S., Prada Moroni, P. G., \& Tordiglione, V. 2002, MNRAS, 334, 193

ESA 1997, $S P-\mathbf{1 2 0 0}$

Gatewood, G., de Jonge, J. K., \& Han, I. 2000, ApJ, 533, 938-943

Gomez, A. E., Luri, X., Mennessier, M. O., Torra, J., \& Figueras, F. 1997, in Hipparcos - Venice '97, ESA SP-402, 207-209

Gomez, A. E., Luri, X., Grenier, S., Figueras, F., North, P., Royer, F., Torra, J., \& Mennessier, M. O. 1998, A\&SA, 336, 953-959

Grenon, M. 2002, in Highlights in Astronomy, 12, 680-683

Hertzsprung, E. 1915, Astronomische Nachrichten 200, 137

Hertzsprung, E. 1947, Annal.Sterrewacht Leiden, 19, 1-85

Jordi, C., Luri, X., Masana, E., Torra, J., Figueras, F., Domingo, A., Gómez, A. E., \& Mennessier, M. O. 2002, Highlights in Astronomy, 12, 684-687

Knapp, G. R., Pourbaix, D., Platais, I., \& Jorissen, A. 2003, A\&A A, 403, 993-1002

Knapp, G., Pourbaix, D., \& Jorissen, A. 2001, A\& A, 371, 222-223

Kovalevsky, J., Falin, J. L., Pieplu, J. L., Bernacca, P. L., Donati, F. et al. 1992, A $\mho A$ A, 258, $7-17$

van Leeuwen, F. 1984, in Galactic and Solar System Optical Astrometry, (ed. L. V. Morisson and G. Gilmore), Cambridge University Press, 223

van Leeuwen, F. 1997, Space Sci. Rev., 81, 201-412

van Leeuwen, F. 1999a, A\&SA, 341, L71-74

van Leeuwen, F. 1999b, in Harmonizing Cosmic Distance Scales in a Post-HIPPARCOS Era, (ed. D.Egret \& A. Heck), ASP Conf. Ser. 167, 52-71

van Leeuwen, F. 2000, in Stellar Clusters and Associations: Convection, Rotation, and Dynamos, (ed. R. Pallavicini, G. Micela, S. Sciortino), ASP Conf. Ser. 198, 85-94

van Leeuwen, F. \& Alphenaar, P. 1982, The Messenger 28, 15-18

van Leeuwen, F., Alphenaar, P., \& Brand, J. 1986, A\&SAS, 65, 309-347

van Leeuwen, F., Alphenaar, P., \& Meys, J. J. M. 1987, A\& AS, 67, 483-489

van Leeuwen, F. \& Evans, D. W. 1998, A\& $A S$, 130, 157-172

van Leeuwen, F. \& Fantino, E. 2003, Space Sci. Rev., 108, 537

van Leeuwen, F. \& Hansen-Ruiz, C. S. 1997, in Hipparcos Venice '97, ed. M. A. C. Perryman and P. L. Bernacca, ESA, SP-402, 689

van Leeuwen, F. \& Penston, M. J. 2003, Space Sci. Rev., 108, 471-497

Lindegren, L. 1995, A\&A, 304, 61-68

Lindegren, L., Høg, E., van Leeuwen, F., Murray, C. A., et al. 1992, A\&A A, 258, 18-30 
Madsen, S., Dravins, D. \& Lindegren, L. 2002, A\& $\mathcal{E}$, 381, 446-463

Makarov, V. V. 2002, AJ, 124, 3299-3304

Makarov, V. V. 2003, AJ, 126, 2408-2410

van der Marel, H. \& Petersen, C. S. 1992 A\&A, 258, 60-69

Munari, U., Dallaporta, S., Siviero, A., Soubiran, C., Fiorucci, M., \& Girard, P. 2004, A\&A, 418, L31-34

Narayanan, V. K. \& Gould, A. 1999, ApJ, 523, 328-339

Pan, X., Shao, M., \& Kulkarni, S. R. 2004, Nature, 427, 326

Percival, S. M., Salaris, M., \& Kilkenny, D. 2003, A\& A, 400, 541-552

Pinsonneault, M. H., Stauffer, J., Soderblom, D. R., King, J. R., \& Hanson, R. B. 1998, ApJ, $\mathbf{5 0 4}, 170$

Pinsonneault, M. H., Terndrup, D. M., Hanson, R. B., \& Stauffer, J. R. 2004, ApJ, 600, 946-959

Pourbaix, D. \& Boffin, H. M. J. 2003, A\& A, 398, 1163-1177

Pourbaix, D. \& Jorissen, A. 2000, A\&AS, 145, 161

Robichon, N., Arenou, F., Mermilliod, J.-C., \& Turon, C. 1999, A\&A A, 345, 471-484

Robichon, N., Lebreton, Y., Turon, C., \& Mermilliod, J.-C. 2000, in Stellar Clusters and Associations: Convection, Rotation, and Dynamos, ASP Conf. Ser. 198, 141-144

Stauffer, J. R., Hartmann, L., Soderblom, D. R., \& Burnham, N. 1984, ApJ, 280, 202-212

Stauffer, J. R., Jones, B. F., Backman, D., Hartmann, L. W., Barrado y Navascués, D., Pinsonneault, M. H., Terndrup, D. M., \& Muench, A. A. 2003, AJ, 126, 833-847

Vasilevskis, S., van Leeuwen, F., Nicholson, W., \& Murray, C. A. 1979, A\&AS, 37, 333-343

\section{Discussion}

FRITz BENEDICT: First of all I would like to apologise for not quoting the smaller number in my paper. There's still a $7 \sigma$ difference, even with the smaller number. The HR Diagram which you showed has a considerable spread in the main sequence. Is some fraction of that due to binaries?

FLOOR VAN LEeUwen: There's far more than the binaries; they are just $\frac{3}{4}$ of a magnitude. There are nearly two magnitudes here, most of which is due to evolution away from the main sequence. It appears for many different reasons that the evolutionary effects are much more severe, in terms of age, on the typical main sequence star, than seems to be represented in the isochrones. The question is, above all, to understand where the difference comes from. The question is not settled. It will be settled when we understand quantitatively where this difference comes from. We get two independent results from the same data, admittedly, but none of them is leading to a parallax of $7.3,7.4$ milli-arcseconds for the Pleiades.

RICKY SMART: Some of the problems that have come up recently with this Pleiades discussion has put the whole of the Hipparcos results into question. There was a paper that actually said this. What's your view on this?

FLOOR VAN LEEUWEN: I do not agree with the results of this paper. I consider that its conclusions are incorrect. 


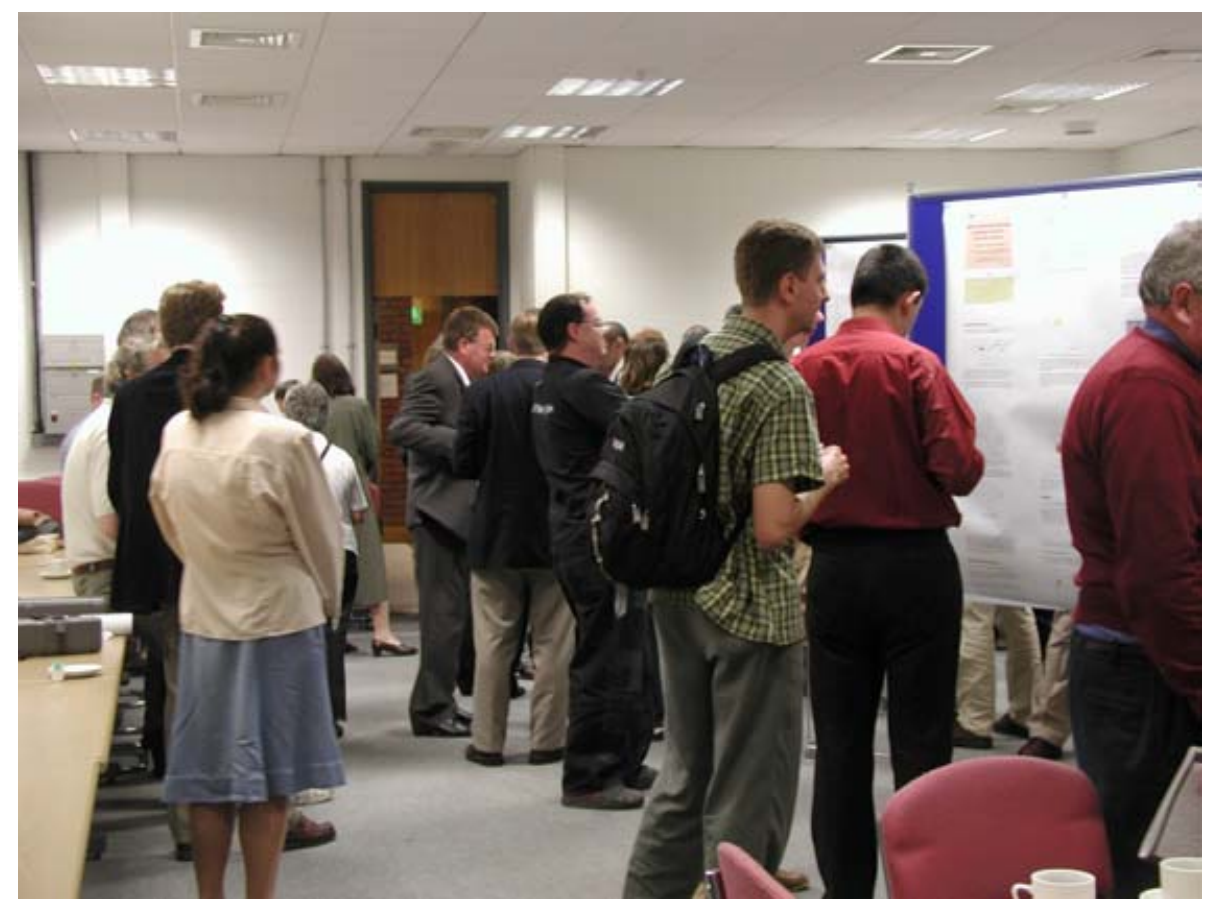

Poster Viewing
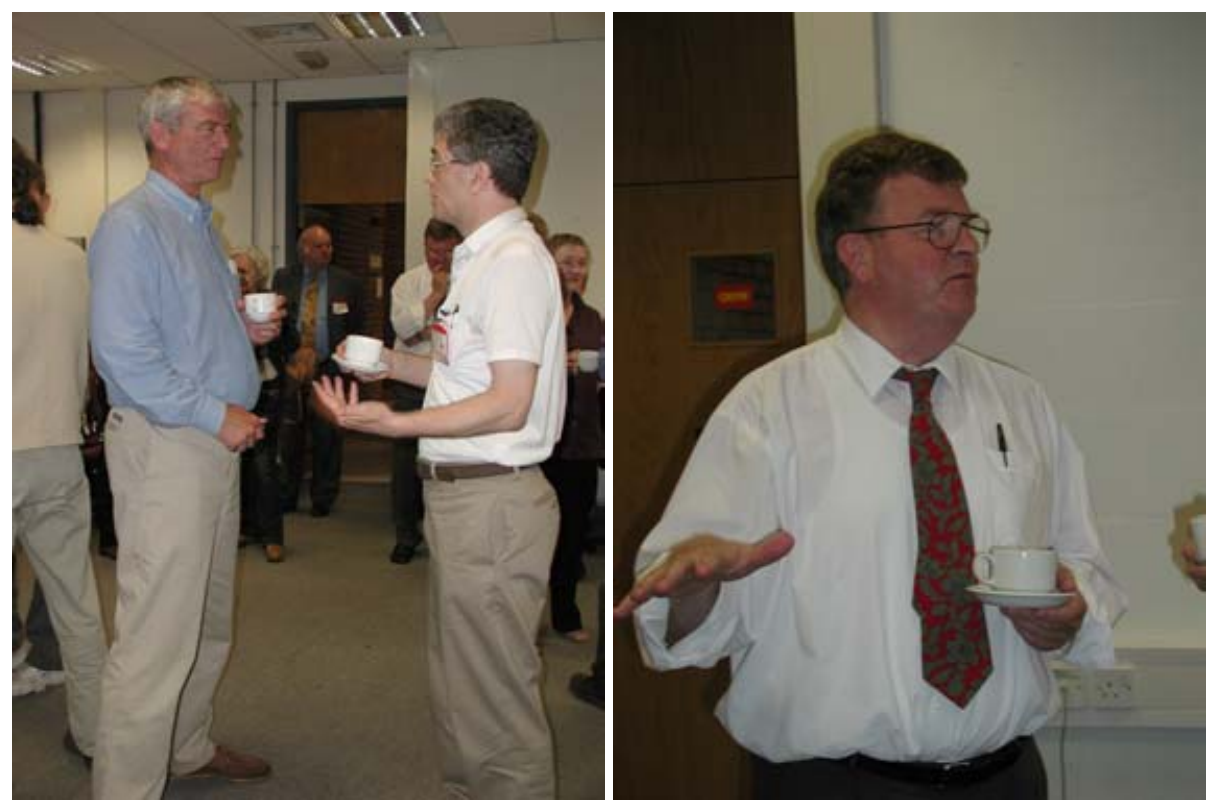

left: Floor van Leeuwen talking to Myles Standish; right: Ed Budding 